\title{
MRI/US fusion prostate biopsy in men on active surveillance: Our experience
}

\author{
Vito Lacetera ${ }^{1}$, Angelo Antezza ${ }^{2}$, Alessio Papaveri ${ }^{2}$, Emanuele Cappa ${ }^{1}$, Bernardino Cervelli ${ }^{1}$, \\ Giuliana Gabrielloni ${ }^{1}$, Michele Montesi ${ }^{1}$, Roberto Morcellini ${ }^{1}$, Gianni Parri ${ }^{1}$, Emilio Recanatini ${ }^{1}$, \\ Valerio Beatrici ${ }^{1}$ \\ ${ }^{1}$ Azienda Ospedaliera Ospedali Riuniti Marche Nord, Division of Urology, Pesaro, Italy; \\ ${ }^{2}$ Università Politecnica delle Marche-Azienda Ospedaliera Ospedali Riuniti Torrette di Ancona, Italy;
}

\begin{abstract}
Summary Aim: The upgrading or staging in men with prostate cancer (PCA) undergoing active surveillance (AS), defined as Gleason score (GS) $\geq 3+4$ or more than 2 area with cancer, was investigated in our experience using the software-based fusion biopsy (FB) Methods: We selected from our database, composed of 620 biopsies, only men on AS according to criteria of John Hopkins Protocol (T1c, $<3$ positive cores, $G S=3+3=6$ ). Monitoring consisted of PSA measurement every 3 months, a clinical examination every 6 months, confirmatory FB within 6 months and then annual FB in all men. The suspicious MRI lesions were scored according to the Prostate Imaging Reporting and Data System (PI-RADS) classification version 2. FB were performed with a transrectal elastic free-hand fusion platform. The overall and clinically significant cancer detection rate was reported. Secondary, the diagnostic role of systematic biopsies was evaluated.

Results: We selected 56 patients on AS with mean age 67.4 years, mean PSA $6.7 \mathrm{ng} / \mathrm{ml}$ and at least one follow-up MRI-US fusion biopsy (10 had 2 or 3 follow-up biopsies). Lesions detected by MRI were: PIRADS-2 in 5, PIRADS-3 in 28, PIRADS-4 in 18 pts and PIRADS-5 in 5 patients. In each MRI lesion, FB with $2.1 \pm 1.1$ cores were taken with a mean total cores of $13 \pm 2.4$ including the systematic cores. The overall cancer detection rate was 71\% (40/56): 62\% (25/40) in target core and $28 \%(15 / 40)$ in systematic core. The overall significant cancer detection rate was $46 \%$ (26/56): $69 \%$ (18/26) in target vs $31 \%(8 / 26)$ in random cores.

Conclusions: The incidence of clinical significant cancer was $46 \%$ in men starting active surveillance, but it was more than doubled using MRI/US Target Biopsy 69\% (18/26) rather than random cores $(31 \%, 8 / 26)$. However, $1 / 3$ of disease upgrades would have been missed if only the targeted biopsies were performed. Based on our experience, MRI/US fusion target biopsy must be associated to systematic biopsies to improve detection of significant cancer, reducing the risks of misclassification.
\end{abstract}

KEY WORDS: Fusion Biopsy; MRI-US guided Fusion Biopsy; Prostate Cancer; Active Surveillance.

Submitted 14 January 2021; Accepted 27 January 2021

\section{INTRODUCTION}

Active surveillance (AS) is currently the most rapidly growing management strategy for men with low risk prostate cancer (PCA). Aim of AS is to defer or avoid treatments preventing the side effects after active treatments (urinary incontinence and erectile dysfunction) (1). Biopsy criteria for AS vary from one protocol to another: in the original AS was offered only for men with small Gleason score (GS) $3+3=6$ PCA according to Epstein criteria of indolent PCA (2-3), but now in few programs the criteria include men with more extensive GS $3+3=6$ lesions and even some with GS 3+4 (5)

Upgrading beyond the low-risk cancer found initially has been reported in 35-45\% during the first year of follow-up using systematic biopsies (SB) $(6,7)$. Early disease upgrading likely indicates that the initial biopsy findings were inaccurate therefore a more accurate characterization of prostate pathologic findings from the beginning of AS (and during follow-up) would be desirable. Magnetic resonance imaging and MRI-US fusion biopsy (FB) has been shown to help characterizing pathologic findings more accurately than SB, leading to improved detection of significant PCA. Use of this new biopsy method has not yet fully evaluated among men undergoing active surveillance (7-9). We present our experience using MRI-US FB in men undergoing AS of PCA.

\section{MATERIALS AND METHOdS}

This retrospective single center study included 620 consecutive patients who underwent FB between May 2016 and January 2019. We selected from our database only patients on AS. All patients had at least one suspicious lesion at mpMRI, that were performed in different centers as it often happens in community setting without a central review. The suspicious lesions were scored according to the PI-RADS classification v.2. FB were performed with Koelis ${ }^{\mathrm{TM}}$ system (Koelis, Meylan, France), using Koelis Trinity ${ }^{\mathrm{TM}}$ platform.Koelis ${ }^{\mathrm{TM}}$ system creates a precise and highly detailed 3D map of the prostate integrating 3D ultrasound, elastic fusion and Organ-Based Tracking ${ }^{\circledR}$. All the biopsies considered in the study were performed with a transrectal approach, as reported in our initial experienceby 3 experienced urologists dedicated to FB (10). Biopsy were performed and specimens collected according to Italian guidelines (11). PCA was considered clinically significant in case of findings of 


\section{Figure 1.}

Fusion + systematic biopsy in a patient on Active Surveillance: 3 targets on a PIRADS 3 lesion in TZa right middle gland and 13 random cores avoiding the previous tracks (blue cores). Results: 2 positive cores GS 3+3 = 6 on target lesion, AS was continued.

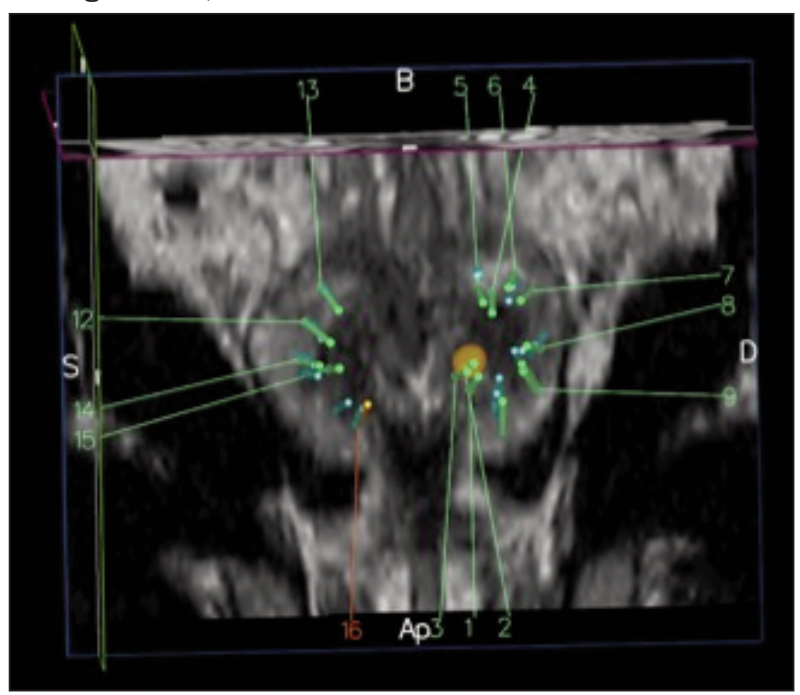

Figure 2.

Anterior-posterior view shows the target core inside the anterior TZ PIRADS 3 lesion at MRI.

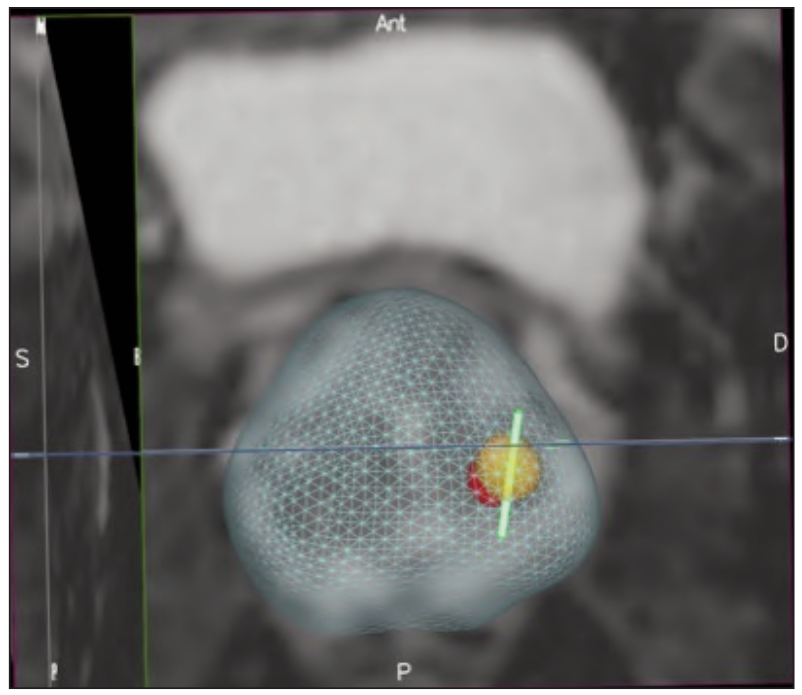

Gleason score $>6$, or more than 2 cores of Gleason score 6 (or more than 1 core outside the target) as suggested by criteria of many AS protocols. The overall and clinically significantcancer detection rate (oCDR, cs CDR) of Koelis $^{\mathrm{TM}}$ system was obtained. Secondary the diagnostic role of additional SB was evaluated (Figures 1-3).

\section{RESULTS}

We selected from our database $56 / 620$ patients on AS with at least 1 follow-up FB. The characteristics of the 56 patients (summarized in Table 1) were: mean age 67.4 years $(C I \pm 8.8)$; mean PSA $6.7 \mathrm{ng} / \mathrm{ml} \pm 3.1$; mean prostate volume $49.2 \pm 21 \mathrm{ml}$. Lesions detected by MRI: PIRADS 2 = 5 pts; PIRADS $3=28$ pts; PIRADS $4=18$
Figure 3.

Confirmatory biopsy in a 54 yo patient on AS: Fusion biopsy (6 target and 6 sistematyc cores) was PCA positive in 3/6 cores (GS $4+3=7$ ). Patient undergone to RARP (Final pathology confirmed a GS $=4+3=7$ pT2RONO).

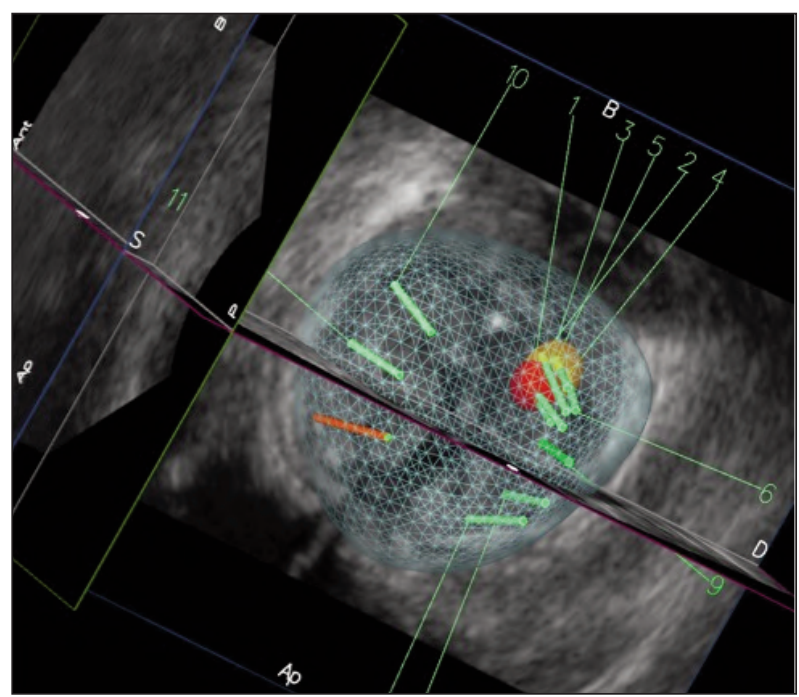

pts; PIRADS $5=5$ pts. 46 pts had only 1 confirmatory FB, 7 pts had 2 follow-up FB, 3 pts had 3 follow-up FB. Mean cores from each MRI target lesion were $2.1 \pm 1.1$; mean total cores were $13 \pm 2.4$. Overall PCA detection rate was 71\% (40/56); overall significant PCA (Gleason Score $>=3+4)$ detection rate was $=46 \%(26 / 56)$; PCA in target core $=62 \%(25 / 40) ;$ PCA in random core $=28 \%$ $(15 / 40)$; significant PCA in target cores $=69 \%(18 / 26)$; significant PCA in systematic core $=31 \%(8 / 26)$ (as summarized in Table 2).

Table 1.

Characteristics of the patients.

\begin{tabular}{|l|c|}
\hline Number of patients & 56 \\
\hline Age (years), mean (Cl) & $67.4 \pm 8.8$ \\
\hline PSA (ng/ml), mean (Cl) & $6.7 \pm 3.1$ \\
\hline Prostate volume (ml), mean (Cl) & $49.2 \pm 21$ \\
\hline PIRADS of targets (maximum score in case of multiple targets) & 5 \\
PIRADS 2 & 28 \\
PIRADS 3 & 18 \\
PIRADS 4 & 5 \\
PIRADS 5 & $2.1 \pm 1.1$ \\
\hline Mean target cores & $13 \pm 2.4$ \\
\hline Mean total cores & \\
\hline
\end{tabular}

Table 2.

Results.

\begin{tabular}{|l|c|}
\hline Characteristic & Number \\
\hline Overall PCA detection rate & $71 \%(40 / 56)$ \\
\hline Significant PCA detection rate & $46 \%(26 / 56)$ \\
\hline PCA detected in target cores & $62 \%(25 / 40)$ \\
\hline PCA in detected in systematic cores & $28 \%(15 / 40)$ \\
\hline Significant PCA detected in target cores & $69 \%(18 / 26)$ \\
\hline Significant PCA detected in systematic cores & $31 \%(8 / 26)$ \\
\hline
\end{tabular}




\section{Discussion}

Among the devices used for FB, Koelis ${ }^{\mathrm{TM}}$ is supported by severalrobust evidences (12-14), showing a CDR ranging from $48 \%$ to $80 \%$.

We compared our results with Nassiri et al. (15), who analyzed 259 men (196 with GS 3+3 and 63 with GS $3+4$ ) who were diagnosed by MRI/US FB (period 20092015) and who underwent subsequent FB for as long as 4 years of AS: $63 \%$ of men with GS 3+4 were upgraded by the third surveillance year, compared with $18.0 \%$ of men with initial GS $3+3(p<0.01)$. Moreover, $97 \%$ of all upgrades (32/33) occurred within an MRI-visible or a tracked site of tumor, rather than a previously-negative systematic site. Jayadevan et al. (17) analyzed men with a new diagnosis of Gleason grade Group (GG) 1 PCA (period 2009-2017). The initial diagnostic biopsy was performed by various methods in community settings and within one year from diagnosis, all the men underwent confirmatory FB. Confirmatory biopsy and all follow-up biopsies were performed using a MRI-guided biopsy system. The end point was a finding of at least GG3 disease during follow-up, which then excluded those patients from active surveillance. Of 332 patients in the total cohort of AS, 114 had normal findings on confirmatory biopsy, 175 had GG1 disease, and 43 had GG2 disease. There were 39 patients (11.7\%) with upgrading to at least GG3 during the study period with $43 \%$ of upgraded cases detected only by target biopsies (TB) and 46\% d only by SB. Thus, if only one biopsy method was implemented, at least $43 \%$ of disease upgrades would have been missed. Similar findings were seen in Improvement in the Detection of Aggressive Prostate Cancer by Targeted Biopsies Using Multiparametric MRI Findings (MRI-FIRST) and the Prospective Assessment of Image Registration in the Diagnosis of Prostate Cancer (PAIREDCAP) trials $(17,18)$.

An analysis of patients undergoing AS by Frye et al. (19) at the National Cancer Institute also found that the combination of SB and TB should be used during AS followup, given that only $30 \%$ of pathologic disease upgrades were identified by SB alone.

The efficacy of the combination of both biopsy techniques has been recently confirmed a by Klots et al. (20) and Ma et al. (21).

In order to reduce side effects of systematic biopsy, several non-invasive strategies has been proposed (22).

The PSA-density, as supported by Roscigno et al. (23), was used with a cut-off $\geq 0.20 \mathrm{ng} / \mathrm{mL}$ to improve the predictive accuracy of mpMRI results for reclassification of patients in AS, whereas a PSAD value $<0.10 \mathrm{ng} / \mathrm{mL}$ identifies a lower risk of harboring clinically significant cancer. Nowadays, the combination of target and SB represents the standard for patients on AS; our study strengthens this recommendation, showing that additional random cores improved the overall CDR of $28 \%$ and clinically significant CDR of $31 \%$.

\section{Conclusions}

FB represents a useful tool to address many of the limitations of contemporary systematic biopsy. According to most recent evidences and our experience, we believe that MRI/US fusion biopsy improve overall cancer detection rate, clinical significant cancer detection rate and risk stratification among men on active surveillance. Our data suggest that confirmatory and follow-up fusion biopsies with MRI guidance when associated to SB provide a more accurate risk assessmentin order to reduce the oncological risks of AS.

\section{References}

1. Cooperberg MR. Active surveillance for low-risk prostate cancer - an evolving international standard of care. JAMA Oncol. 2017; 3:1398-1399.

2. Gasparrini S, Cimadamore A, Mazzucchelli R, et al. Pathology and molecular updates in tumors of the prostate: towards a personalized approach. Expert Rev Mol Diagn. 2017; 17:781-789.

3. Montironi R, Santoni M, Mazzucchelli R, et al. Prostate cancer: from Gleason scoring to prognostic grade grouping. Expert Rev Anticancer Ther. 2016; 16:433-40.

4. Mazzucchelli R, Galosi AB, Lopez-Beltran A, et al. Pathological issues in biopsy specimens of men with prostate cancer eligible for active surveillance. Arch Ital Urol Androl. 2014; 30;86:314-8.

5. Klotz L. Active surveillance for prostate cancer: overview and update. Curr Treat Options Oncol. 2013; 14:97-108.

6. Dinh KT, Mahal BA, Ziehr DR et al. Incidence and predictors of upgrading and upstaging among 10,000 contemporary patients with low risk prostate cancer. J Urol. 2015; 194:343-349.

7. Barrett T, Haider MA. The emerging role of MRI in prostate cancer active surveillance and ongoing challenges. AJR Am J Roentgenol. 2017; 208:131-139.

8. Elkhoury FF, Simopoulos DN, Marks LS. Targeted prostate biopsy in the era of active surveillance. Urology. 2018; 112:12-19.

9. Kasivisvanathan V, Rannikko AS, Borghi M, et al. PRECISION Study Group Collaborators. MRI-targeted or standard biopsy for prostate-cancer diagnosis. N Engl J Med. 2018; 378:1767-1777.

10. Lacetera V, Cervelli B, Cicetti A, et al. MRI/US fusion prostate biopsy: our initial experience. Arch Ital Urol Androl. 2016; 88:296-299.

11. Fandella A, Scattoni V, Galosi A, et al. Italian Prostate Biopsies Group: 2016 Updated Guidelines Insights. Anticancer Res. 2017; $37: 413-424$.

12. Baco E, Rud E, Eri LM, et al. A randomized controlled trial to assess and compare the outcomes of two-core prostate biopsy guided by fused magnetic resonance and transrectal ultrasound images and traditional 12-core systematic biopsy. Eur Urol. 2016; 69:149-156.

13. Ukimura O, Gross ME, de Castro Abreu AL, et al. A novel technique using three-dimensionally documented biopsy mapping allows precise re-visiting of prostate cancer foci with serial surveillance of cell cycle progression gene panel. Prostate. 2015; 75:863-71.

14. Mozer P, Rouprêt M, Le Cossec C, et al. First round of targeted biopsies using magnetic resonance imaging/ultrasonography fusion compared with conventional transrectal ultrasonography-guided biopsies for the diagnosis of localised prostate cancer. BJU Int. 2015; 115:50-7.

15. Nassiri N, Margolis DJ, Natarajan S, et al. Targeted biopsy to detect Gleason score upgrading during active surveillance for men with low versus intermediate risk prostate cancer. J Urol. 2017; 197:632-639.

16. Jayadevan, et al. Magnetic resonance imaging-guided confirma- 
tory biopsy for initiating active surveillance of prostate cancer JAMA Netw Open. 2019; 2:e1911019.

17. Rouvière O, Puech P, Renard-Penna R, et al. MRIFIRST Investigators. Use of prostate systematic and targeted biopsy on the basis of multiparametric MRI in biopsy-naive patients (MRIFIRST): a prospective, multicentre, paired diagnostic study. Lancet Oncol. 2019; 20:100-109.

18. Elkhoury FF, Felker ER, Kwan L, et al. Comparison of targeted vs systematic prostate biopsy in men who are biopsy naive: the Prospective Assessment of Image Registration in the Diagnosis of Prostate Cancer (PAIREDCAP) study JAMA Surg. 2019; 154:811-818.

19. Frye TP, et al. Magnetic resonance imaging-transrectal ultrasound guided fusion biopsy to detect progression in patients with existing lesions on active surveillance for low and intermediate risk prostate cancer. J Urol. 2017; 197:640-646.
20. Klotz L, Loblaw A, Sugar L, et al. Active Surveillance Magnetic Resonance Imaging Study (ASIST): results of a randomized multicenter prospective trial. Eur Urol. 2019; 75:300-309.

21. Ma TM, Tosoian JJ, et al. The role of multiparametric magnetic resonance imaging/ultrasound fusion biopsy in active surveillance. Eur Urol. 2017; 71:174-180.

22. Cimadore A, Scarpelli M, Raspolini MR, et al. Prostate cancer pathology: What has changed in the last 5 years. Urologia 2020; 87:3-10.

23. Roscigno M, Stabile A, Lughezzani $G$, et al The use of multiparametric magnetic resonance imaging for follow-up of patients included in active surveillance protocol. Can PSA density discriminate patients at different risk of reclassification? Clin Genitourin Cancer. 2020; 18:e698-e704.

\section{Correspondence}

Vito Lacetera, MD (Corresponding Author)

vlacetera@gmail.com

Emanuele Cappa, MD

cappa.emanuele@gmail.com

Bernardino Cervelli, MD

Bernardo.Cervelli@ospedalimarchenord.it

Giuliana Gabrielloni, MD

Giuliana.Gabrielloni@ospedalimarchenord.it

Michele Montesi, MD

Michele.Montesi@ospedalimarchenord.it

Roberto Morcellini, MD

Roberto.Morcellini@ospedalimarchenord.it

Gianni Parri, MD

Gianni.Parri@ospedalimarchenord.it

Emilio Recanatini, $M D$

Emilio.Recanatini@ospedalimarchenord.it

Valerio Beatrici, MD

Valerio.Beatrici@ospedalimarchenord.it

Urologist, Azienda Ospedaliera Ospedali Riuniti Marche Nord, Pesaro,

Division of Urology A.O. Ospedali Riuniti Marche Nord,

Piazzale Cinelli 4, 61121 Pesaro (Italy)

Angelo Antezza, MD

angelo.antezza@yahoo.it

Alessio Papaveri, $M D$

alessio.papaveril@gmail.com

Resident in Urology, Università Politecnica delle Marche-Azienda Ospedaliera

Ospedali Riuniti Torrette di Ancona (Italy) 